\title{
Electronic Structure of Spin-Chain Compounds: Common Features
}

\author{
U. Schwingenschlögl and C. Schuster \\ Institut für Physik, Universität Augsburg, 86135 Augsburg, Germany
}

\begin{abstract}
The incommensurate composite systems $\mathrm{M}_{14} \mathrm{Cu}_{24} \mathrm{O}_{41}(\mathrm{M}=\mathrm{Ca}, \mathrm{Sr}, \mathrm{La})$ are based on two fundamental structural units: $\mathrm{CuO}_{2}$ chains and $\mathrm{Cu}_{2} \mathrm{O}_{3}$ ladders. We present electronic structure calculations within density functional theory in order to address the interrelations between chains and ladders. The calculations account for the details of the crystal structure by means of a unit cell comprising 10 chain and 7 ladder units. It turns out that chains and ladders can be treated independently, which allows us to introduce a model system based on a reduced unit cell. For the $\mathrm{CuO}_{2}$ chains, we find two characteristic bands at the Fermi energy. Tight binding fits yield nearest and next-nearest neighbour interactions of the same order of magnitude.
\end{abstract}

PACS numbers: 71.10.Pm: Fermions in reduced dimensions, 71.20.-b: Electron density of states and band structure of crystalline solids, 74.72.-h: Cuprate superconductors

Keywords:

The isostructural spin-chain compounds $\mathrm{M}_{14} \mathrm{Cu}_{24} \mathrm{O}_{41}(\mathrm{M}=\mathrm{Ca}, \mathrm{Sr}, \mathrm{La})$ have been subject of intensive research in recent years, mainly due to their rich phase diagram and close relations to the high- $\mathrm{T}_{c}$ cuprates. Their incommensurate crystal structures consist of planes of quasi one-dimensional $\mathrm{CuO}_{2}$ chains stacked alternately with planes of two-leg $\mathrm{Cu}_{2} \mathrm{O}_{3}$ ladders. The orientation of these chains and ladders defines the crystallographical $c$-axis, where the lattice constants of the two subsystems satisfy in a good approximation $10 c_{\text {chain }} \approx 7 c_{\text {ladder }}[1,[2$. The copper ions are intrinsically hole doped with nominal $\mathrm{Cu}$ valence +2.25 for both the $\mathrm{Ca}$ and the $\mathrm{Sr}$ compound. However, the ladders contain less holes than the chains. The resulting $\mathrm{Cu}$ valence of +2.75 for the chain sites corresponds to a quarter filled one-dimensional band [3]. Optical conductivity as well as x-ray absorption experiments suggest that substitution of $\mathrm{Ca}$ for $\mathrm{Sr}$ induces a transfer of holes from the chains back to the ladders [4]. On La substitution the intrinsic doping decreases, reaching the undoped state with nominal $\mathrm{Cu}$ valence +2.00 for $\mathrm{La}_{6} \mathrm{Ca}_{8} \mathrm{Cu}_{24} \mathrm{O}_{41}$.

In the case of $\mathrm{Sr}_{14} \mathrm{Cu}_{24} \mathrm{O}_{41}$ the $\mathrm{CuO}_{2}$ chains are non-magnetic with a spin gap of about $130 \mathrm{~K}\left[\underline{5}\right.$ ] and the $\mathrm{Cu}_{2} \mathrm{O}_{3}$ ladders show a charge density wave in a wide temperature range [6, 7]. In contrast, for Ca rich samples antiferromagnetic ordering is found in the chains [8, 9] and the ladders become superconducting under pressure [10]. This strong doping dependence of both the charge and the magnetic order [11, 12, 13, 14] is accompanied by only minor modifications of the crystal structure [15, 16, 17]. From the theoretical point of view, the magnetic properties of undoped two-leg $\mathrm{S}=1 / 2$ ladders have been analyzed be means of a Heisenberg model including cyclic ring exchange [18]. Possible effects of hole doping are investigated in [19] A large spin gap makes the $\mathrm{Cu}_{2} \mathrm{O}_{3}$ ladder planes magnetically inert. On the other hand, the magnetic phase diagram of the $\mathrm{CuO}_{2}$ chains is quite complicated. Ferromagnetism, as expected for $\mathrm{Cu}-\mathrm{O}-\mathrm{Cu}$ bond angles of approximately $90^{\circ}$ in the chains, is realized in La rich systems. The origin of the observed intrachain antiferromagnetic order for a quarter filled $\mathrm{Cu}$ band, however, led to controversial discussions in the literature [17, 20, 21]. Understanding of the doping dependence of both the charge order and the spin gap is likewise far from complete.

Electronic structure calculations applying density functional theory and the local density approximation have been performed by Müller et al. 22] for the isostructural $\mathrm{Cu}_{2} \mathrm{O}_{3}$ ladders in $\mathrm{SrCu}_{2} \mathrm{O}_{3}$ and by Arai et al. 23] for a simplified $\mathrm{Sr}_{14} \mathrm{Cu}_{24} \mathrm{O}_{41}$ unit cell. Probably because of a high demand on CPU time, state-of-the-art band structure calculations accounting for the details of the crystal structures are absent so far. In the present letter we aim at filling this gap in order to address the interplay between the chain and ladder subsystems and to identify electronic features common to all compounds due to the specific crystal structure. Moreover, we introduce a model system capturing the relevant properties of the $\mathrm{CuO}_{2}$ chains for various compounds and levels of hole doping. By this model system, we identify the electronic states at the Fermi level and study intrachain nearest and next-nearest neighbour interactions in the framework of a tight binding model. As we account for the decisive structural ingredients, we obtain, as far as we know for the first time, realistic insights into the electronic structure of the $\mathrm{CuO}_{2}$ chains in spin-chain compounds.

Our first principles band structure calculations for the full $\mathrm{Sr}_{14} \mathrm{Cu}_{24} \mathrm{O}_{41}$ unit cell are based on the scalar-relativistic augmented spherical wave (ASW) method 24], which has proven to be suitable for dealing with unit cells comprising a large number of atomic sites 25, 26, 27]. As structural input we apply the crystallographical data due to a recent structural refinement by Gotoh et al. [2]. A previous investigation of the crystal structure by Ukei et al. [1] seems to suffer from low quality samples. Since the $\mathrm{Sr}_{14} \mathrm{Cu}_{24} \mathrm{O}_{41}$ unit cell contains two formula units, we have to deal with 28 strontium, 48 copper, and 82 oxygen atomic spheres. Furthermore, 146 additional augmentation spheres are necessary in order to represent the correct shape of the crystal potential in the large voids of the open crystal structure. The basis sets taken into account in the secular matrix therefore comprise $\mathrm{Sr} 5 s, 5 p, 4 d, \mathrm{Cu} 3 d, 4 s, 4 p$, and $\mathrm{O} 2 s, 2 p$ orbitals, as well as states of the additional augmentation spheres. Brillouin zone integrations are performed using an 

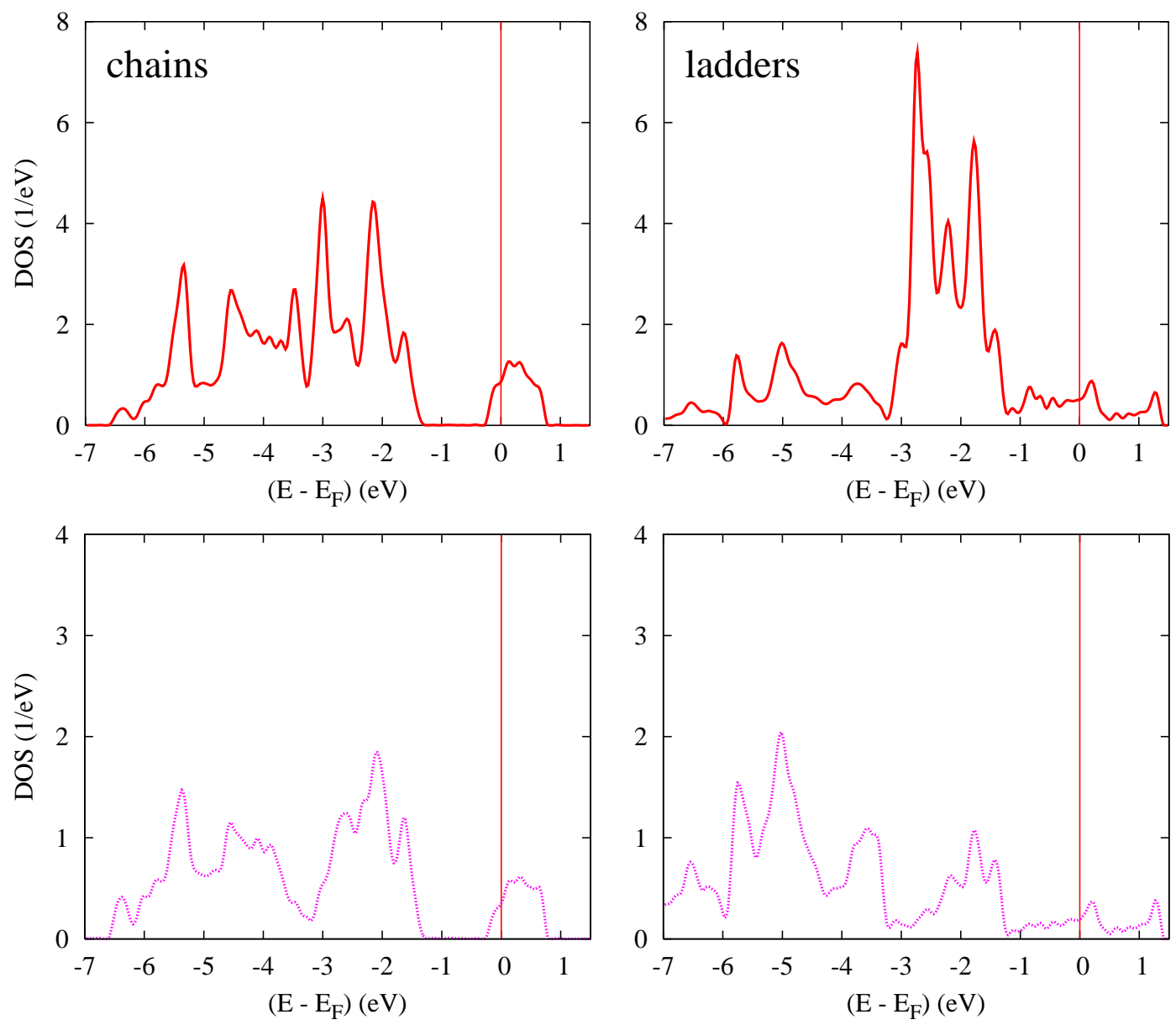

FIG. 1: Partial $\mathrm{Cu} 3 d$ (first row) and $\mathrm{O} 2 p$ (second row) densities of states (per atom) for the chain (left) and ladder (right) subsystems. The results are based on the full $\mathrm{Sr}_{14} \mathrm{Cu}_{24} \mathrm{O}_{41}$ unit cell.

increasing number of finally $72 \mathbf{k}$-points in the irreducible wedge. The Vosko-Wilk-Nusair parametrization is used for the exchange-correlation functional.

The model system for the $\mathrm{CuO}_{2}$ chain discussed subsequently comprises 6 atomic sites. It is studied via band structure calculations using the WIEN2k program package, which is a popular full-potential linearized augmented plane wave (LAPW) code [28]. To obtain reliable results, we apply basis sets of 18135 plane waves and $\mathbf{k}$-meshes with up to 108 points in the irreducible wedge of the Brillouin zone. Whereas the $\mathrm{Cu} 3 p$ and $\mathrm{O} 1 s$ orbitals are treated as semi-core states, the valence states consist of $\mathrm{Cu} 3 d, 4 s, 4 p$ and $\mathrm{O} 2 s, 2 p$ orbitals. The data are based on the Perdew-Burke-Ernzernhof scheme for the exchange-correlation functional.

Figure 1 displays partial $\mathrm{Cu} 3 d$ densities of states (DOS) resulting from our calculations for the full $\mathrm{Sr}_{14} \mathrm{Cu}_{24} \mathrm{O}_{41}$ unit cell. The $\mathrm{Cu} 3 d \mathrm{DOS}$ is seperated into contributions due to the $\mathrm{CuO}_{2}$ chains (left) and $\mathrm{Cu}_{2} \mathrm{O}_{3}$ ladders (right), and normalized with respect to the number of contributing sites. We take into account the structural details of the compound by means of a unit cell comprising 10 chain and 7 ladder units along the crystallographical $c$-axis. This unit cell gives rise to an excellent approximation to the incommensurate crystal structure [2]. Note that different lengths of the chain and ladder units in principle lead to slight periodical tiltings, which do not affect our conclusions and therefore are not considered here.

Both for the chains and ladders there is a finite DOS at the Fermi energy in fig. 1. contradicting the experimentally observed non-metallic state of $\mathrm{Sr}_{14} \mathrm{Cu}_{24} \mathrm{O}_{41}$. In order to reproduce the insulating behaviour, it would be necessary to include electronic correlations beyond the local density approximation. However, for our purpose of studying the interplay between the chains and ladders such local interactions are not of interest. Electronic states in the energy range of fig. 1 1 are composed of $\mathrm{Cu} 3 d$ and $\mathrm{O} 2 p$ orbitals (almost exclusively), which indicates strong $\mathrm{Cu}-\mathrm{O}$ hybridization both in the chains and in the ladders. As expected, this resembles the findings for the simplified unit cell of Arai et al. 23]. The same is true for the gross shapes of the chain and ladder DOS curves. While the ladder DOS is spread over the whole energy range from $-7 \mathrm{eV}$ to $1.5 \mathrm{eV}$, the chain DOS shows a distinct band with a width of $1 \mathrm{eV}$ near the 

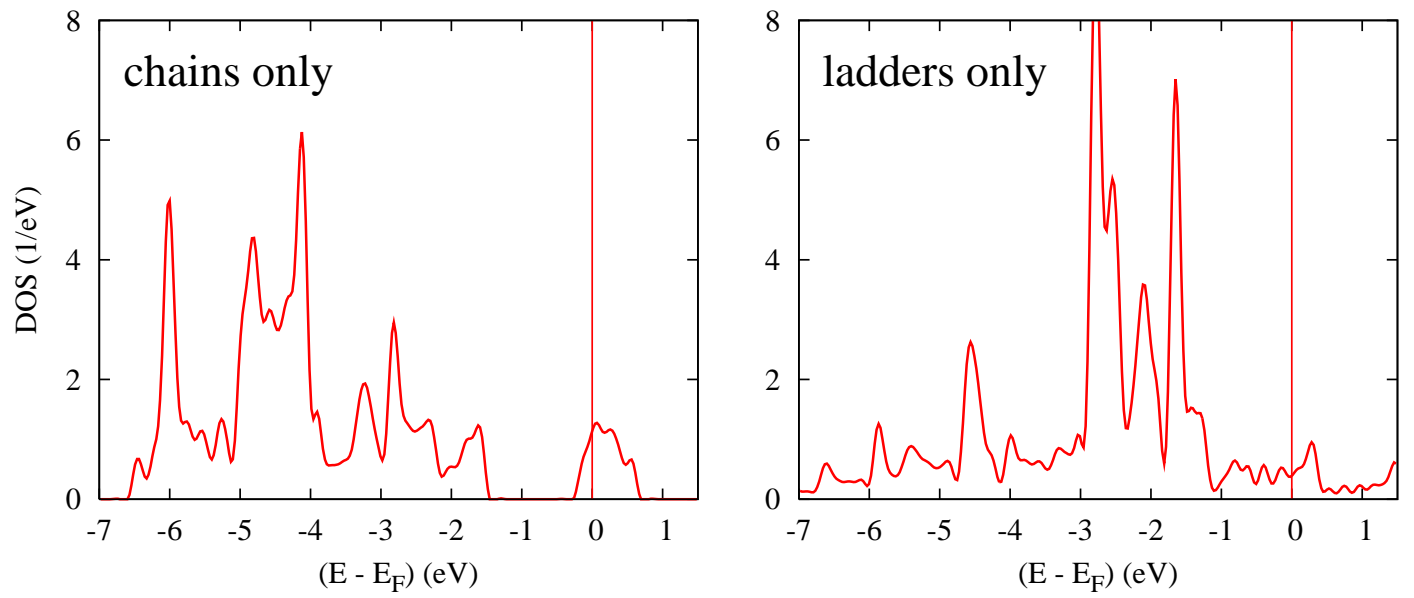

FIG. 2: Partial $\mathrm{Cu} 3 d$ densities of states for chain (left) and ladder (right) copper sites. The results are based on $\mathrm{Sr}_{14} \mathrm{Cu}_{24} \mathrm{O}_{41}$ unit cells where either the chain or the ladder substructure was removed. As concerns the vicinity of the Fermi level, the curves resemble almost perfectly the findings for the chain and ladder copper sites in the full $\mathrm{Sr}_{14} \mathrm{Cu}_{24} \mathrm{O}_{41}$ unit cell, respectively, compare fig. 1 .
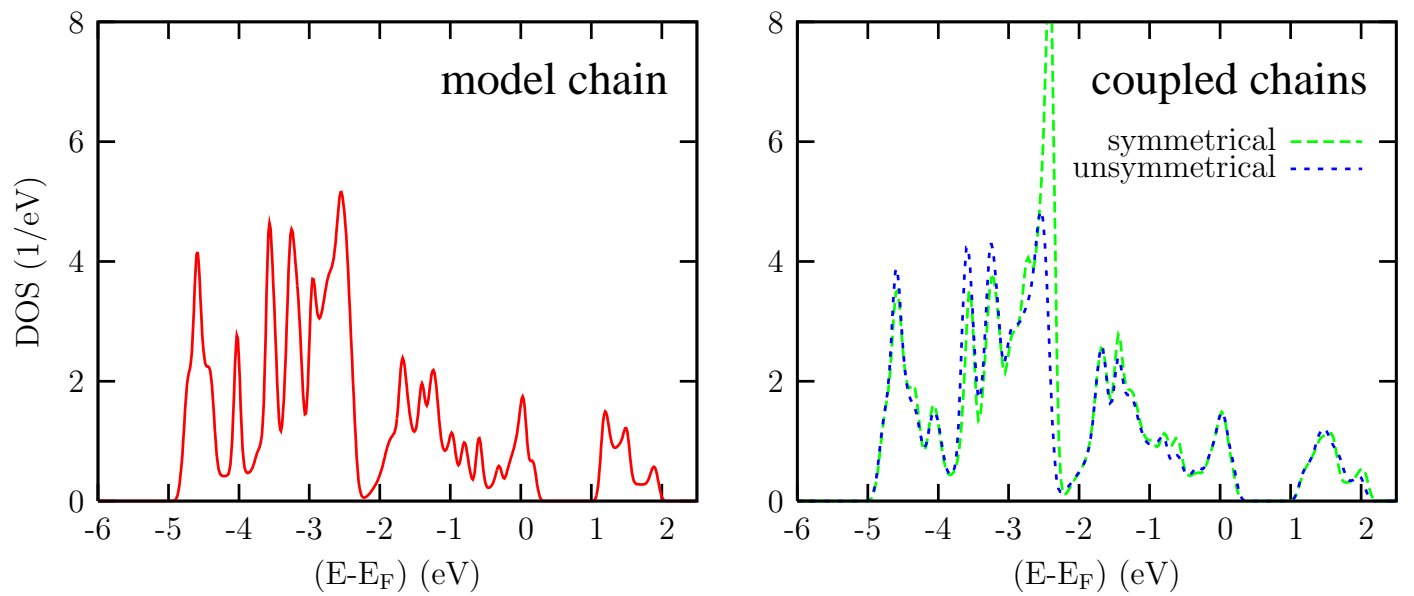

FIG. 3: Partial $\mathrm{Cu} 3 d$ densities of states for a model $\mathrm{CuO}_{2}$ chain (left) and two coupled $\mathrm{CuO} 2$ chains (right), arranged symmetrically and unsymmetrically. The states are subject to a rigid band shift of about $1.5 \mathrm{eV}$ as compared to the real system.

Fermi level, separated by a gap of likewise $1 \mathrm{eV}$ from the other valence bands. This band is close to quarter filling, as to be expected, and hence subject to a large variety of possible ordering processes of the charge and spin degrees of freedom [29].

Turning to the question about the interrelations between the chain and ladder subsystem, the $\mathrm{Sr}_{14} \mathrm{Cu}_{24} \mathrm{O}_{41}$ unit cell is modified as follows: (a) We remove either the chain or the ladder substructure and (b) replace Sr by K. Since the $\mathrm{M}$ ions in $\mathrm{M}_{14} \mathrm{Cu}_{24} \mathrm{O}_{41}$ act almost exclusively as charge donors, the latter allows us to maintain the correct doping when removing one of the substructures. For the full unit cell, formally one electron is transferred from each $\mathrm{Sr}$ site to both the chain and the ladder subsystem. The same amount of charge transfer is achieved for the half-filled unit cell via the potassium, because hybridization effects between $\mathrm{Sr} / \mathrm{K}$ and $\mathrm{Cu} / \mathrm{O}$ orbitals are negligible. Figure 2 displays DOS curves for the chains only configuration on the left and for the ladders only configuration on the right side. In both cases the findings resemble the corresponding curves of fig. 2 as concerns the total width of the valence bands as well as the gross shape of the DOS. In the vicinity of the Fermi energy even the details of the DOS are identical. In particular, for the $\mathrm{CuO}_{2}$ chains, the band width and the filling of the distinct band around the Fermi level hardly alter.

To conclude, since the above argumentation does not depend on the specific choice of the donor ion M, we find that spin-chain compounds $\mathrm{M}_{14} \mathrm{Cu}_{24} \mathrm{O}_{41}$ are well described in terms of three largely independent subsystems: The $\mathrm{CuO}_{2}$ chains, the $\mathrm{Cu}_{2} \mathrm{O}_{3}$ ladders, and the electron donor system of $\mathrm{M}$ ions, which separates chains and ladders. 

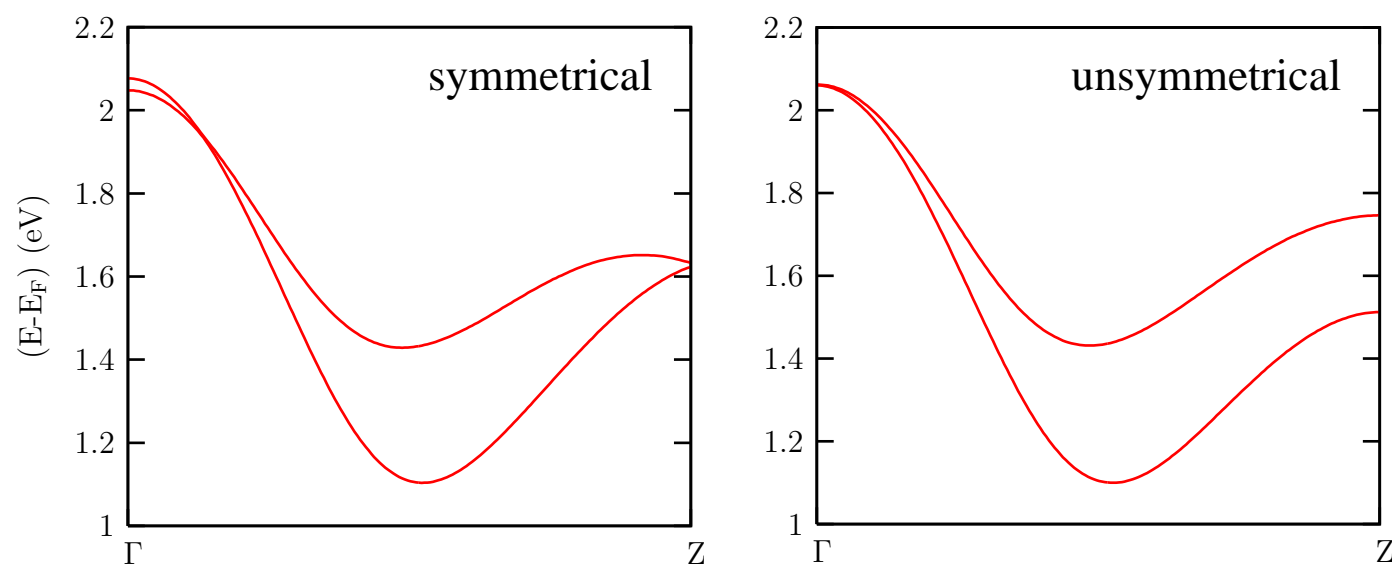

FIG. 4: Electronic band structure ( $\mathrm{Cu} 3 d$ bands) for coupled $\mathrm{CuO}_{2}$ chains, arranged symmetrically (left) and unsymmetrically (right). The results of tight-binding fits are summarized in tabel 1

band 1 , symm. band 2, symm.|band 1 , unsymm. band 2 , unsymm.

\begin{tabular}{c|c|c|c|c}
\hline$t_{1}(\mathrm{eV})$ & -0.115 & -0.105 & -0.135 & -0.080 \\
$t_{2}(\mathrm{eV})$ & -0.183 & -0.098 & -0.165 & -0.115 \\
$\epsilon_{0}(\mathrm{eV})$ & 1.485 & 1.645 & 1.460 & 1.675
\end{tabular}

TABLE I: Tight-binding parameters for the $\mathrm{Cu} 3 d$ bands of fig. 4

Hybridization between atomic orbitals belonging to different subsystems can be neglected because the valence states of the $\mathrm{M}$ ions are almost fully depopulated. As a consequence, we attribute charge transfer between chains and ladders when replacing $\mathrm{Sr}$ by isoelectronic Ca [3, 4] to simultaneous modifications of the crystal structure.

Since we have established that the structural subsystems of spin-chain compounds can be treated independently, we address the electronic features of the $\mathrm{CuO}_{2}$ chains in the following via a model system based on a reduced unit cell. For convenience, we choose the Euklidean $z$-axis as the chain axis and use the interatomic distances and bond angles of $\mathrm{Sr}_{14} \mathrm{Cu}_{24} \mathrm{O}_{41}$. Each $\mathrm{Cu}$ site thus is connected to two symmetrical $\mathrm{O}$ sites in both chain directions, where the bond lengths are $1.89 \AA$ in one and $1.87 \AA$ in the other direction. The related $\mathrm{O}-\mathrm{Cu}-\mathrm{O}$ bond angles are $85.5^{\circ}$ and $86.7^{\circ}$, respectively. Neighbouring $\mathrm{Cu}$ atoms are separated by $2.75 \AA$. In order to prepare for the symmetry analysis of the $\mathrm{Cu}$ bands, we align the $\mathrm{CuO}_{2}$ chains in the Euklidean $x z$-plane. As a consequence, $\mathrm{Cu} 3 d_{x z}$ orbitals mediate the main part of the $\mathrm{Cu}-\mathrm{O}$ overlap. We study both a single $\mathrm{CuO}_{2}$ chain, for which the unit cell comprises just one $\mathrm{CuO}_{2}$ unit, and two coupled chains. The latter allows us to address the influence of relative shifts between adjacent chains with respect to the chain axis, which distinguish specific spin-chain compounds. For $\mathrm{Ca}_{13.6} \mathrm{Sr}_{0.4} \mathrm{Cu}_{24} \mathrm{O}_{41}$ [9, 15] the chains are shifted by half their intrachain $\mathrm{Cu}-\mathrm{Cu}$ distance, for example, while the shift is only $30 \%$ of the $\mathrm{Cu}-\mathrm{Cu}$ distance for $\mathrm{Sr}_{14} \mathrm{Cu}_{24} \mathrm{O}_{41}$. We compare these two cases, which we call the symmetrically and unsymmetrically arranged chains, respectively.

Figure 3 summarizes the results of our LAPW model calculations, showing partial $\mathrm{Cu} 3 d$ densities of states for a single $\mathrm{CuO}_{2}$ chain on the left side and two coupled $\mathrm{CuO}_{2}$ chains on the right side. All three DOS curves resemble the essential features of the ASW data for the real system, see figs. 1 and 2, We obtain a widespread structure with a width of about $5.2 \mathrm{eV}$ at lower energies and a structure extending over a range of $1 \mathrm{eV}$ at higher energies. However, comparison with the ASW findings results in a rigid band shift of slightly less than $1.5 \mathrm{eV}$ to higher energies. This shift traces back to the fact that the $\mathrm{M}$ ions are not taken into account in the model system, see the previous discussion. In the following we hence are interested in the electronic states in the energy range from about $1 \mathrm{eV}$ to $2 \mathrm{eV}$. As expected, these states are mainly due to the antibonding combination of $\mathrm{Cu} 3 d_{x z}$ and both $\mathrm{O} 2 p_{x}$ and $2 p_{z}$ orbitals. Contributions of other states amount to less than $0.1 \%$ of the total DOS at the Fermi energy. In order to obtain further insight into the electronic features of the $\mathrm{CuO}_{2}$ chains, we turn to the band structure underlying the DOS curves of fig. 3.

Because the $\mathrm{CuO}_{2}$ chains run parallel to the $z$-axis of our unit cell, fig. 4 depicts the $\mathrm{Cu} d_{x z}$ bands in the corresponding direction of the orthorhombic Brillouin zone, i.e. between the high symmetry points $\Gamma$ and $Z$. As the length of a $\mathrm{CuO}_{2}$ unit amounts to $c_{\text {chain }}=2.75 \AA$, we can write $\mathbf{k}_{\Gamma}=(0,0,0)$ and $\mathbf{k}_{Z}=\left(0,0,2 \pi / c_{\text {chain }}\right)$. Reasonable fitting of 
the bands is possible by assuming a the tight-binding dispersion of the form

$$
\epsilon\left(k_{z}\right)=\epsilon_{0}-2 t_{1} \cos \left(k_{z} c_{\text {chain }}\right)-2 t_{2} \cos \left(2 k_{z} c_{\text {chain }}\right),
$$

where $k_{z}$ is the $z$-component of the reciprocal lattice vector and $\epsilon_{0}$ is the band center. Moreover, $t_{1}$ and $t_{2}$ denote the nearest and next-nearest neighbour hopping parameters along the chains, respectively. The outcome of the tight-binding fits for the bands of fig. [ is given in table I Beyond our one-dimensional model (1), influence of finite interchain hopping can be identified in the band structure for the symmetrical configuration, but is not relevant for our further conclusions. In contrast to Arai et al. [23], we find two $\mathrm{Cu} 3 d_{x z}$ bands, corresponding to the two coupled chains. We attribute this difference to inadequate crystallographical data entering the former band calculation. Importantly, we obtain for both $\mathrm{Cu} 3 d_{x z}$ bands nearest and next-nearest neighbour couplings of the same order of magnitude, independent of shifts between neighbouring $\mathrm{CuO}_{2}$ chains. Despite the $90^{\circ} \mathrm{Cu}-\mathrm{O}-\mathrm{Cu}$ intrachain bond angles, strong nearest neighbour interaction therefore appears to be typical of the whole class of spin-chain compounds. As the calculated magnitudes of the nearest and next-nearest neighbour coupling are common to all systems, we conclude that changes in the magnetic ordering on replacing $\mathrm{Ca}$ by La result almost exclusively from the modified filling of the $\mathrm{Cu} 3 d_{x z}$ bands.

In summary, we have studied the electronic structure of $\mathrm{Sr}_{14} \mathrm{Cu}_{24} \mathrm{O}_{41}$ by means of density functional theory. Taking into account the details of the crystal structure, we have addressed the interrelations between the structural subsystems the spin-chain compounds $\mathrm{M}_{14} \mathrm{Cu}_{24} \mathrm{O}_{41}\left(\mathrm{M}=\mathrm{Ca}\right.$,Sr,La) are composed of. Hybridization between the $\mathrm{CuO}_{2}$ chains, the $\mathrm{Cu}_{2} \mathrm{O}_{3}$ ladders, and the electron donor $\mathrm{M}$ ions is found to be negligible. Since these subsystems consequently can be treated individually, we have analyzed a simplified model system for the $\mathrm{CuO}_{2}$ chains to identify features common to all spin-chain compounds. It turns out that two characteristic $\mathrm{Cu} 3 d_{x z}$ bands at the Fermi energy dominate the electronic and magnetic properties of the chains. The filling of these bands distinguishes different compounds. Tightbinding fits prove that nearest and next-nearest neighbour interactions in the $\mathrm{CuO}_{2}$ chains are of the same order of magnitude, which applies to the whole class of spin-chain systems.

\section{Acknowledgments}

We thank U. Eckern for helpful discussions and the Deutsche Forschungsgemeinschaft for financial support (SFB 484).

[1] Ukei K., Shishido T. Fukuda T. Acta Cryst.B50199442.

[2] Gotoh Y., Yamaguchi I., Takahashi Y., Akimoto J., Goto M., Onoda M., Fujino H., Nagata T. Akimitsu J. Phys. Rev. B682003224108.

[3] Nücker N. et al. Phys. Rev. B62200014384.

[4] Osafune T., Motoyama N., Eisaki H. Uchida S. Phys. Rev. Lett.7819971980.

[5] Matsuda M. Katsumata K. Phys. Rev. B53199612201.

[6] Gorshunov B., Haas P., Rõõm T., Dressel M., Vuletić T., Korin-Hamzić B., Tomić S., Akimitsu J. Nagata T. Phys. Rev. B662002060508.

[7] Gozar A., Blumberg G., Littlewood P.B., Dennis B.S., Motoyama N., Eisaki H. Uchida S. Phys. Rev. Lett.912003087401.

[8] Nagata T., Fujino H., Akimitsu J., Nishi M., Kakurai K., Katano S., Hiroi M., Sera M. Kobayashi N. J. Phys. Soc. Jpn.6819992206.

[9] Isobe M., Onoda M., Ohta T., Izumi F., Kimoto K., Takayama-Muromachi E., Hewat A.W. Ohoyama K. Phys. Rev. B62200011667.

[10] Uehara M., Nagata T., Akimitsu J., Takahashi H., Môri N. Kinoshita K. J. Phys. Soc. Jpn.6519962764.

[11] Carter S.A., Batlogg B., Cava R.J., Krajewski J.J., Peck W.F. Jr. Rice T.M. Phys. Rev. Lett.7719961378.

[12] Ammerahl U., Büchner B., Colonescu L., Gross R. Revcolevschi A. Phys. Rev. B6220008630.

[13] Braden M., Etrillard J., Gukasov A., Ammerahl U. Revcolevschi A. Phys. Rev. B692004214426.

[14] Kataev V., Choi K.-Y., Grüninger M., Ammerahl U., Büchner B., Freimuth A. Revcolevschi A. Phys. Rev. B642001104422.

[15] Ohta T., Izumi F., Onoda M., Isobe M., Takayama-Muromachi E. Hewat A.W. J. Phys. Soc. Jpn.6619973107.

[16] Matsuda M., Katsumata K., Osafune T., Motoyama N., Eisaki H., Uchida S., Yokoo T., Shapiro S.M., Shirane G. Zarestky J.L. Phys. Rev. B56199714499.

[17] Klingeler R. Spin- und Ladungsordnung in Übergangsmetalloxiden - Thermodynamische und magnetische Untersuchungen, Dissertation (RWTH Aachen, 2003).

[18] Nunner T.S., Brune P., Kopp T., Windt M. Grüninger M. Phys. Rev. B662002180404.

[19] Nishimoto S., Jeckelmann E. Scalapino D.J. Phys. Rev. B662002245109.

[20] Gellé A. Lepetit M.-B. Eur. Phys. J. B43200529. 
[21] Klingeler R., Büchner B., Choi K.-Y., Kataev V., Ammerahl U., Revcolevschi A. Schnack J. Phys. Rev. B732006014426.

[22] Müller T.F.A., Anisimov V., Rice T.M., Dasgupta I. Saha-Dasgupta T. Phys. Rev. B571998R12655.

[23] Arai M. Tsunetsugu H. Phys. Rev. B561997R4305.

[24] Eyert V. Int. J. Quantum Chem.7720001007.

[25] Schwingenschlögl U., Eyert V. Eckern U. Europhys. Lett.612003361.

[26] Schwingenschlögl U. Eyert V. Ann. Phys. (Leipzig)132004475.

[27] Schwingenschlögl U. Schuster C. Chem. Phys. Lett.4322006245.

[28] Blaha P., Schwarz K., Madsen G., Kvasicka D. Luitz J. WIEN2k, An Augmented Plane Wave + Local Orbitals Program for Calculating Crystal Properties (TU Wien, 2001).

[29] Schuster C. Schwingenschlögl U. Phys. Rev. B, in press (arXiv:cond-mat/0701235). 